Man kann die Hypothese aufstellen, dass durch irgend eine Schädlickeit, welche im Blut kreist, die Resorption des Knorpels verzögert und vor Allem die Bildung von Knochensubstanz behindert wird. Die in unserem Falle vorhandenen Nekrosen lassen sich vielleicht so deuten, dass bei verstärkter Einwirkung jener Schädlichkeit die Gewebe so sehr beeinträchtigt werden, dass ein Absterben derselben eintritt.

\title{
$\mathbf{X X}$. \\ Ueber die im Malaischen Archipel vorkommenden Malaria-Erreger nebst einigen Fiebercurven
}

\author{
von \\ Dr. Max Glogner, \\ Stadtgeneesher in Samarang (Java). \\ Hierzu Taf. X.-XI.
}

Während in den letzten Jahren die Erreger der MalariaFieber verschiedener Tropengegenden beschrieben wurden, fehlt bis jetzt eine einigermaassen genaue Mittheilung über den MalariaParasiten aus dem Malaischen Archipel, einem der Hauptheerde der Malaria-Erkrankungen. Ich möchte mit folgenden Zeilen diese Lücke ausfüllen und an der Hand einzelner klinisch beobachteter Fälle über die bereits aus anderen Tropengegenden bekannten, sowie über zwei von mir selbst gefundene Erreger berichten, von denen der eine bereits früher in diesem Archiv Gegenstand einer näheren Beschreibung geweseu ist.

Im Malaischen Archipel kommen nach meinen Beobachtungen 4 verschiedene Malaria-Parasiten vor, welche ich auf den beifolgenden Tafeln wiedergebe. Die Krankheitsfälle, bei welchen diese Parasiten gefunden wurden, zeigten klinisch bisweilen die grösste Aehnlichkeit, während sie verschiedene Erreger behor- 

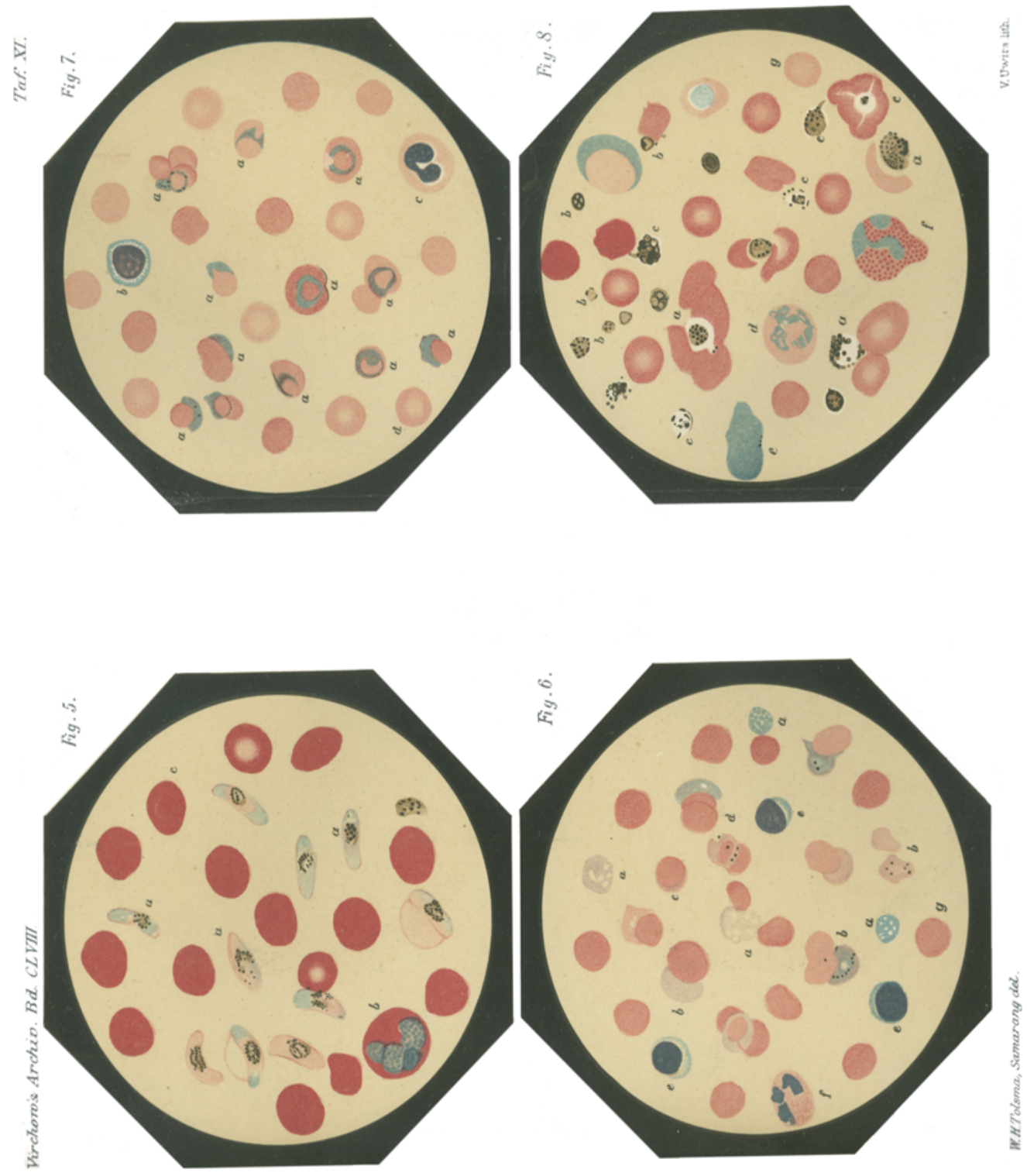
bergten; andere waren durch denselben Erreger entstanden und zeigten doch klinisch grosse. Unterschiede.

Der erste dieser Malaria-Erreger ist der in Europa und anderen tropischen Gegenden vorkommeude Tertian-Parasit, der sich in den rothen Blutkörperchen unter Aufblähung und Abblassen derselben mit Pigmentbildung entwickelt. Bei der Sporulation, wie dieselbe auf Tafel X Fig. 1 durch die Zieman'sche Chromatinfärbung verdeutlicht wird, bilden sich 16-20 Sporen. - Dieser Tertian-Parasit veranlasst zum Theil unsere Tertianfieber, sowie, wenn er in demselben Individuum in verschiedenen Entwickelungs-Stadien vorkommt, die Quotidian-Fieber. So ist in Figur 1 ausser den Theilungsformen noch eine zweite kleinere Parasiten-Colonie zu sehen. Die Präparate, welche diese Abbildung lieferten, entstammen dem Blute eines Chinesen, welcher an einer Tertiana duplex litt, und dessen Temperaturcurve in Tabelle 1 (S. 446) gezeichnet ist.

Toa Lie Siek war seit 18 Tagen in Samarang. Vor vier Jahren wanderte er nach Java ein und wohnte erst in dem gesunden Djorja. Er giebt an, seit 10 Tagen an Fieber zu leiden, welches täglich um 11 Uhr Morgens mit Kältegefühl anfängt; wäbrend der Fieberzeit bat er Kopfschmerzen. Die Milz ist leicht vergrössert, andere Erscheinungen sind nicht vorhanden. Am 12. März, Mittags $12 \mathrm{Uhr}$, bei beginnendern Anfall, wurde das Fingerblut untersucht. Siebentägige Gaben von $1 \mathrm{~g}$ Chinin brachten das Fieber zum Verschwinden.

Etwas ernster gestaltete sich folgender Fall, der demselben Tertian-Erreger seine Entstehung verdankte:

Die Javanin Kasmina wurde Ende iSeptember wegen gonorrboischer Uretbritis ins Krankenbaus aufgenommen, sie litt daran bereits mehrere Monate, batte aber in den letzten Jahren kein Fieber gebabt. Am 21. December klagte sie über Kopfschwerzen und Fieber, and gab an, dass sie bereits seit 10 'Tagen an leichtem Fieber litte; die Temperatur am 21. Dezember war Morgens um $8 \mathrm{Ubr} 37,4^{\circ} \mathrm{C}$., Mittags $5 \mathrm{Uhr} 40,4^{\circ} \mathrm{C}$. Die Kranke gab an, mit den täglich wiederkehrenden Fieberanfällen ein. Gefühl von Steifbeit in den Unterschenkeln, Schmerzen in der Herzgegend, Ameisenlaufen in den Unterarmen und Unterschenkeln, Kurzathmigkeit, sowie ein Vollgefübl in der Magengegend zu verspüren. An beiden Unterschenkeln war Jeichtes Oedem vorbanden. Der Händedrack war, besonders rechts, stark herabgesetzt, Urin enthält Eiweiss, seine Menge betrug von 21.-22. Decbr. $150 \mathrm{ccm}$, die Farbe war rotbbraun; er enthielt mehrere Cylinder und Epithelien. Das Herz war nach rechts bis zum rechten Sternalrand vergrösserst, systoliscbe Gerăuscbe am linken Sternalrand, Puls in Ruhe 128 p. m., Athmung 36 p.m., Druck 
Tabelle 1.

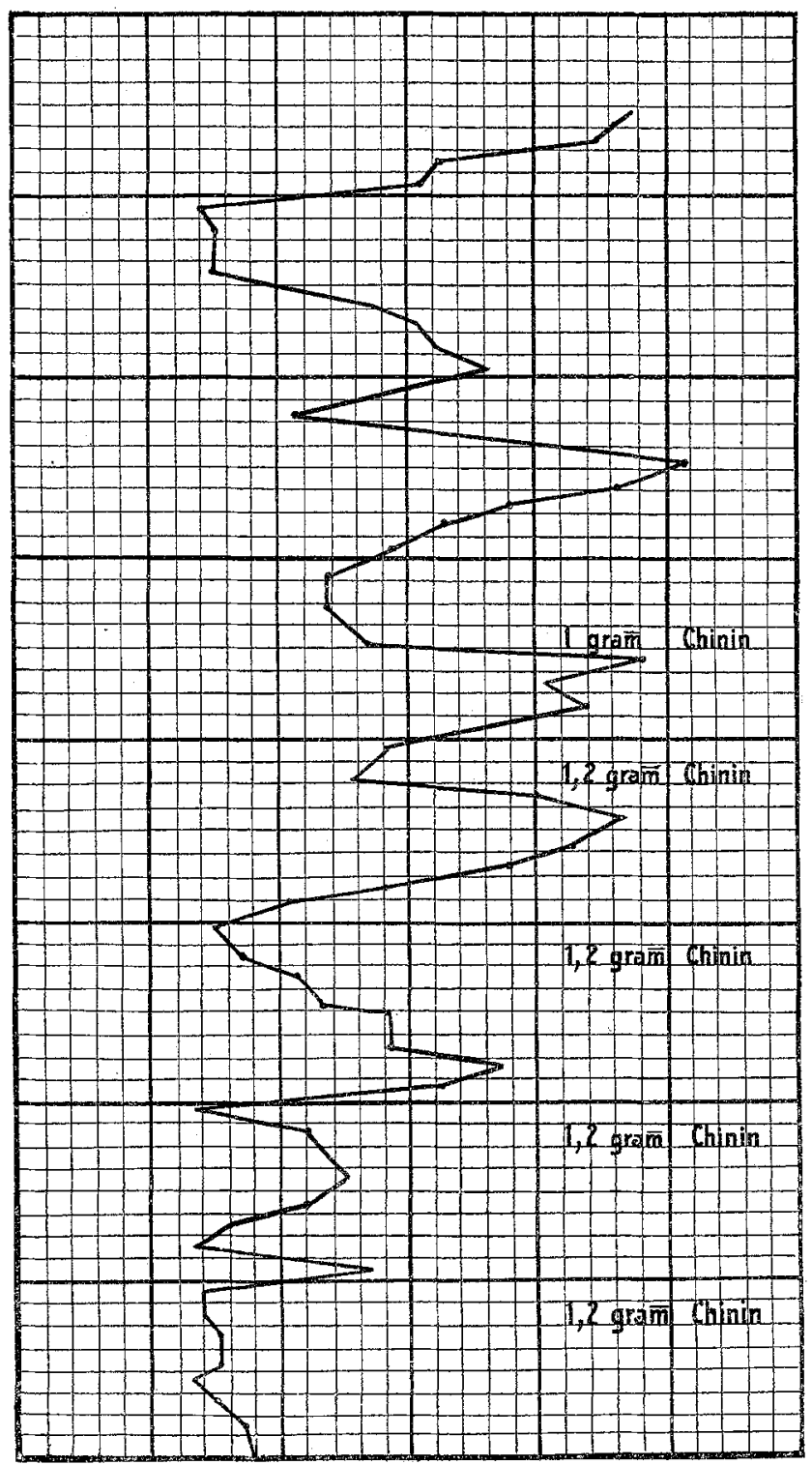


mit dem von Basch'schen Manometer. gemessen, ergab für Radialis dextra $175 \mathrm{~mm}$, Rad. sinistra $185 \mathrm{~mm}$, Femoralis dextra $190 \mathrm{~mm}$, Femoralis sinistra $200 \mathrm{~mm}$. Empfindungs-Qualitäten überall vorhanden. Achilles- und PatellarReflexe aufgehoben, Dorsalflexion beieer Füsse berabgesetzt; die Plantarflexion und die seitlichen Bewegungen der Füsse wurden träge ausgeführt, der Gang der Kranken war schwerfällig und steif. Sie bekam am 21. December ein Laxans, Nitroglycerin und täglich 1 gr Chinin. Vom24. December an war sie bereits fieberfrei, die Kurzathmigkeit, die Schmerzen in Armen, Beinen und Herzgegend, sowie das Ameisenlaufen liessen bald nach. Am 30. December wurde der Blutdruck aufgenommen: Radial. dextra $150 \mathrm{~mm}$, sinistra $160 \mathrm{~mm}$, Femoralis dextra $130 \mathrm{~mm}$, sinistra $200 \mathrm{~mm}$. Das Herz war am 30 . December nicht mehr vergrössert; Athmung 20 p. m., Pals 96 p. m. Die Kranke blieb fieberfrei bis zum 28. Januar; an diesem und dem folgenden Tage bekaın sie kurze Fieberanfälle, ohne $Z_{w}$ eifel ein Recidiv des ersten Tertianfiebers. Die Urinmenge nahm unter Milchdiät allmäblich zu, das Eiweiss schwand, Puls und Athmung schwankten im Januar, Februar und März 2wischen 90 und 110 p. m., bezw. 20-28 p. m. bei normaler Temperatur. Die motorischen Störungen liessen allmählich nach. Die Kranke wurde im Mai gebeilt entlassen.

Dieser Fall unterstützt die früher in diesem Archiv ausgesprochene Behauptung, dass die Malaria-Erreger die Beri-BeriKrankheit hervorzubringen im Stande sind. - Interessant und bemerkenswerth ist die auffallend günstige Wirkung des Nitroglycerin auf die Herzvergrösserung, die ohne $Z$ weifel durch das die Krankheitserreger tödtende Chinin kräftig unterstützt wurde. Ich habe diesen günstigen Einfluss des Nitroglycerin auf das Herz bei der vaso-constrictorischen Form der BeriBeri wiederholt beobachtet; die vorstehende Beobachtung liefert einen weiteren Beweis für die Anschauung, dass die Dilatation des Herzens, besonders die des rechten, durch eine Zusammenziehung der Lungen-Blutgefässe verursacht sein kann. - Dass eine solche Gefässspannung in einzelnen Gefässen bei Kamina vorhanden war, liess der erhöhte Druck in der Femoralis vermuthen. Taf. X, Fig. 2 stammt vom Fingerblut dieses Falles. -

Der zweite im Malaischen Archipel vorkommende MalariaErreger ist ein schwach pigmentirter, endoglobulär sich entwickelnder Organismus, wie ihn Taf. X Figur 3 darstellt; er ist kleiner als der vorige, und bildet nur wenige Sporen, die sich in dem im Allgemeinen nur wenig alterirten Blutkörperchen von einander ablösen; bei $A$ in Figur 3 ist eine derartige Theilungsform wiedergegeben. 
Tabelle 2.

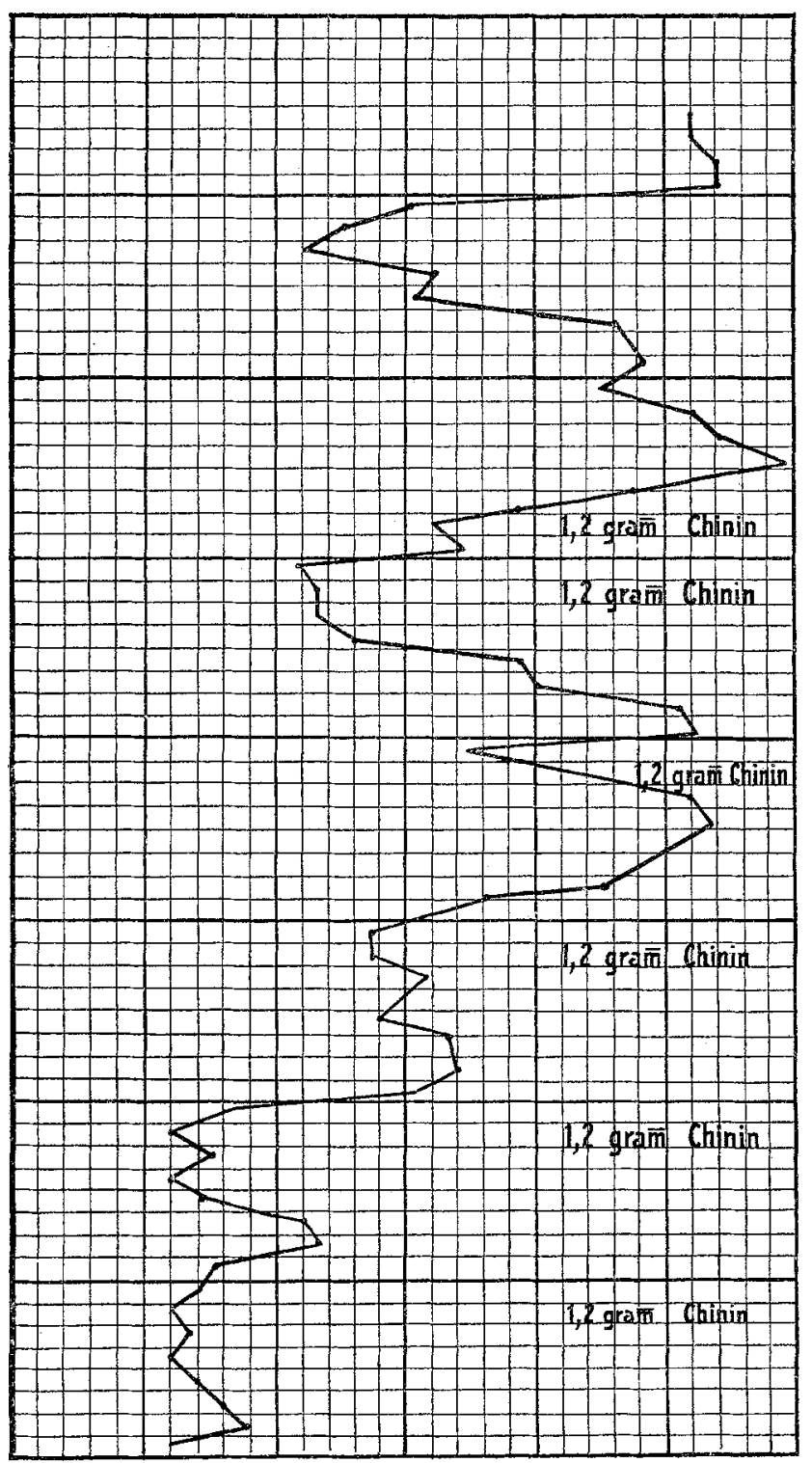


Der Fall, der mir Figur 3 lieferte, war der 20jährige Chinese Lauw Soo Sing. Er gab an, seit einem Monat an täglicb wiederkehrenden Fiebern zu leiden, welche jeden Abend um $7 \mathrm{Ubr}$ mit Schüttelfrost anfingen und um 8 Uhr unter Schweissausbruch endeten; während der äbrigen Zeit des Tages blieb er fieberfrei. In der Fieberzeit fühlte er überall heftige Sehmerzen und Schwere in den oberen und unteren Extremitäten, sowie Kurzathmigkeit; seit 3 Wochen waren die Unterschenkel gelähmt, nach 5 tägigem Fieber konnte er ohne Hülfe nicbt mehr laufen. Vor dem Fieber war er vollstăndig gesund. Die Temperatur war bei dem ersten Besuch um $8 \frac{1}{2}$ Uhr Morgens 37,20 O. Die Bewegung heider Füsse war rollständig aufgehoben, die der Unterschenkel schwerfällig. Der Händedruck war stark herabgesetzt, Sensibilitäts-Störungen fehlten, Achilles- und Patellar-Reflexe aufgehobon. Patient batte täglieh einmal guten Stuhlgang, Urinabscheidung gut, Eiweiss nicht vorhanden. Der Puls betrug bei oben angegebener Temperatur von $37,2{ }^{\circ}$ C. 120 p. m., Athmung 24 p. m. Das Herz war bis zur Mitte des Sternum vergrössert, an der Mitralis systolische Geräusche. Milz palpabel, weich, nach oben reichte die Dämpfung bis zur 6. Rippe. Abends besuchte ich den Patienten zum zweiten Male in seiner Wobnung, und fand Temperatur $37,7^{\circ} \mathrm{C}$., Puls 140 p. m.; es wurden einige Fingerblut-Präparate angefertigt. Um $7 \frac{1}{2}$ Uhr war die Temperatur $39,3^{\circ} \mathrm{C}$. Patient erbielt denselben Abend 1 g Chinin, die folgenden 6 Tage dieselbe Dosis, das Fieber kebrte nicht zurück, zugleich waren Schmerzen und Kurzathmigkeit verschwunden. Die Läbmungen gingen unter elektrischer Behandlung und kräftiger Diäı zuräck.

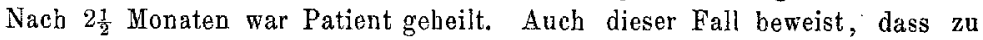
den Ursachen der Beri-Beri die Malaria-Erreger gehören.

Der eben beschriebene und in Figur 3 dargestellte Parasit war ohne $Z$ weifel von quotidianer Entwickelung; in einer grossen Zahl von Präparaten, welche untersucht wurden, fanden sich Parasiten von derselben Grösse; einzelne spärliche, etwas früher gereifte Exemplare, die sich zur Sporulation anschickten, sowie einzelue Jugendformen zeigte nur der bereits um 6 Uhr beginnende Anfall. Dieser Parasit kommt im Allgemeinen selten zur Beobachtung. -

Der dritte der hier vorkommenden Malaria-Erreger ist der in anderen Tropengegenden, häufig in Italien bei den Herbstund Sommerfiebern beobachtete Ringelparasit, von R. Koch als der eigentliche Tropen-Malaria-Erreger bezeichnet, dessen Sporulation ich, ebenso wie andere Beobachter, im Fingerblut nicht beobachten konnte. Die grössten im Fingerblat vorkommenden Ringelparasiten sind von der Grösse $\frac{1}{3}$ eines rothen Blutkörpers. Er kommt im Malaischen Archipel sehr bäufig zur Beobachtung 
und bringt Fieber hervor, welche, wie R. Koch zuerst nachwies, von tertianem Typus sind. Tabelle 1 (S. 446) und 2 (S. 448) liefern hierfür Beweise. -

Gewissermaassen als Anhang zu der Beschreibung des letzten Malaria-Erregers möchte ich die halbmondförmigen Gebilde erwähnen, welche hier im Allgemeinen nicht allzu häufig beobachtet, aber in einzelnen Fällen in grosser Anzahl im Blut vorhanden sind. Figur 4 bringt diese Gebilde zur Anschauung von folgendem Krankheitsfall:

Der Javane Tomerto wurde am 16. November in Krankenhause aufgenommen. Er theilte nur mit, dass er seit 8 Tagen an Fiebern litte, welche jeden Tag um 3 Uhr Mittags anfingen und um 12 Uhr Nachts endeten. Er klagte über ein Gefühl von Schwere im Kopf, Appetit gut, Stublgang regelmässig, Milz leicht vergrössert. Am 16., 17. und 18. November hatte er leichte Mittagsfieber, die Temperatur äberstieg $39,5^{\circ}$ C. nicht; im Fingerblut, welches am 18. November Mittags um $12 \mathrm{Uhr}$ untersucht wurde, wurde eine grosse Anzahl der Sichelformen, sonst aber keiner der andern Parasiten gefunden. Patient erhielt 8 Tage lang täglich $1 \mathrm{~g}$ Chinin; das Fieber schwand am 3. Tage der Chinin-Behandlung, am 8. Tage waren nur noch spärliche Sichelformen nachzuweisen. Patient verliess bald das Krankenhaus und bonnte leider nicht weiter beobachtet werden.

Ich wage es aus der Beobachtung von 10 Fällen nicht mit Sicherheit anzugeben, ob dieser Parasit eine quotidiane oder tertiane Entwickelung besitzt; da die Sporulations-Formen, sowie die grösseren Parasiten mit Vorliebe im Milzblut sich aufzuhalten pflegen und die Bestimmung des Fiebertypus unter solchen Verhältnissen aus der Fingerblut-Untersuchung mit grossen Schwierigkeiten verbunden ist. Die kleinen und mittelgrossen Formen erwiesen sich als Plasmaringe, die fast die Grösse eines rothen Blutkörperchens erreichten. Sie liegen auf oder an rothen Zellen; die eine Hälfte der Parasiten befindet sich bisweilen unter der betreffenden rothen Blutzelle, und dann hat es den Anschein, als ob diese Gebilde eine Halbmondform besässen. Im Allgemeinen ist der Kern bei den jungen Formen schwer zu erkennen, bei den grossen und den Theilungsformen erscheint er als runder oder als eine Anzahl runder weisser Flecke, wie die Figur 6 vom Milzblut des Kertodjojo darstellt. Eine Chromatinfärbung ist mir niemals gelungen, wohl eine nicht allzu starke Färbung des Plasma durch Methylenblau. Dieser Parasit färbt sich ohne $Z$ weifel schwerer, als die anderon erwähnten 
Tabelle 3.

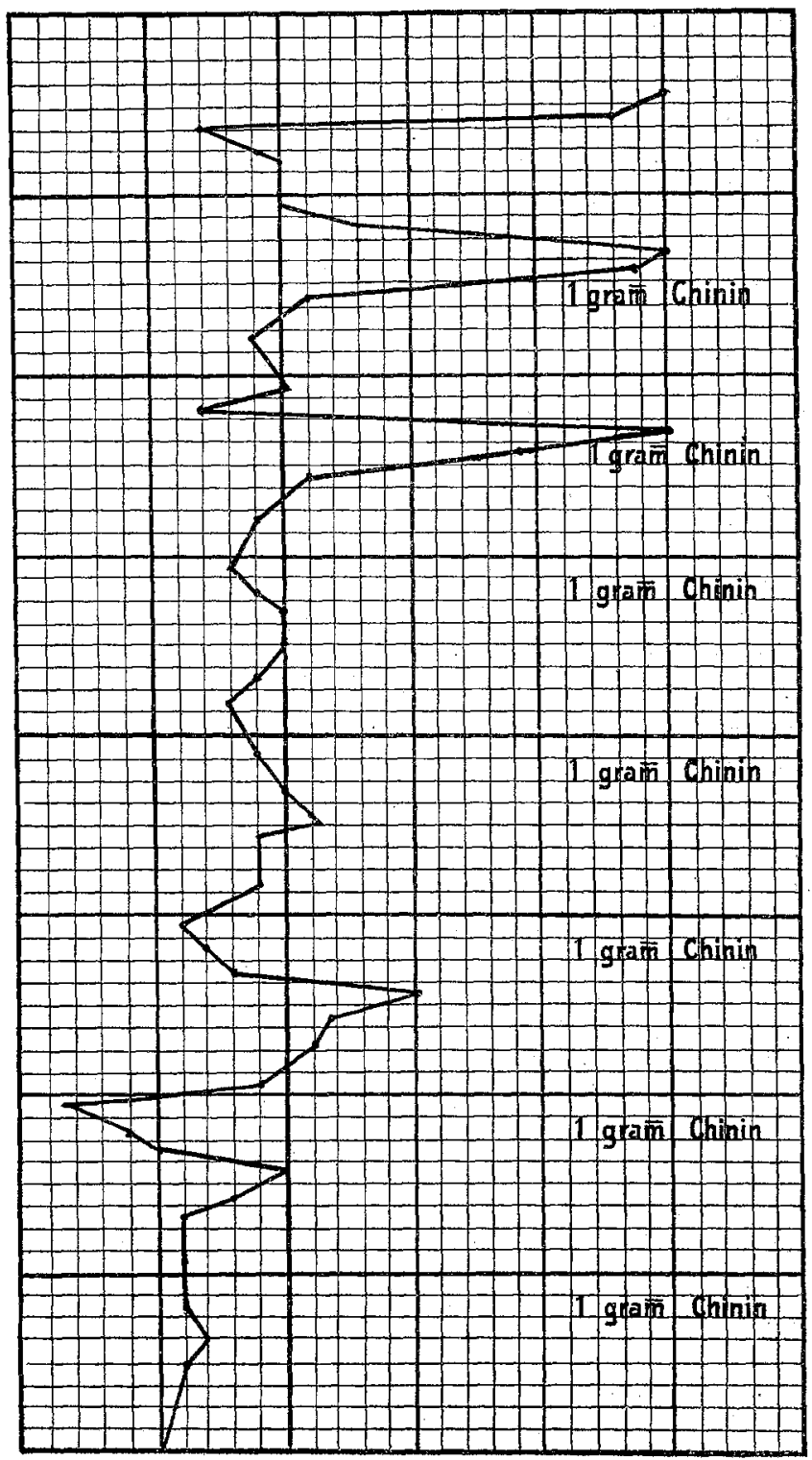


Malaria-Erreger. Ich fand denselben mittels der Zieman'schen Chromatinfärbung, nachdem ich die Präparate eine Stunde in eine 1 pCt. Methylenblau-Lösung mit einer 0,1 pCt. Eosinlösung im Verhältuiss von 1:6 gelegt hatte. Wenn man nun bedenkt, dass das Plasma der anderen Malaria-Erreger mit derselben Farbstofflösung in viel kürzerer Zeit, $\frac{1}{4}-\frac{1}{2}$ Stunde, um vieles intensiver sich färbt, so darf man wohl den Schluss ziehen, dass dieses unpigmentirte Plasmodium zum Methylenblau ein geringeres Anziehungsvermögen besitzt, als die anderen. Was dasselbe aber ganz besonders von den anderen unterscheidet, ist der Mangel an Pigment; weder die Theilungs-, noch die jungen Formen zeigen Pigment. Bei einzelnen gefärbten Exemplaren bemerkt man bisweilen ein körniges Plasma, welches bei nicht allzustarker Vergrösserung eine Pigmentbildung vortäuschen kann.

Figur 4 stammt von dem Javanen Kertodjojo. Sein Milzblut wurde am 16. Juni Mittags 5 Uhr untersucht. Patient theilte mir mit, dass er bereits seit 10 Tagen an Fieber litte, welches Tag und Nacht anhalte; er klagte über Kopfschmerzen. Die Milz war bis zur Nabelhöhe vergrössert. Nacb täglichen Chinin-Dosen von $1 \mathrm{~g}$ trat bald Entfieberung ein, wie dies Tabelle 4 mit der Temperaturcurve zeigt. -

\section{Schwerer war folgender Fall;}

Tjitronello, ein junger Javane, war aus den Goldminen von Celebes krank nach Java zurückgekehrt. Er batte 9 Tage an Fieber gelitten, und es hatte sich zugleich mit dem Fieber eine Lähmung der oberen und unteren Extremitäten eingestellt. Vor $2 \frac{1}{2}$ Monaten wurde er ins hiesige Krankenbaus aufgenommen, Fieber wurde nicht mehr beobachtet. Die einzigen Störungen, die nachgewiesen werden konnten, bestanden in Parese der Unterschenkelund Vorderarm-Muskeln; Herz gesund, Milz nicht vergrössert. Unter elektrischer Behandlung gingen die motorischen Störungen zurück, sodass er fast geheilt war, als plötzlich am 17. Juni hobes Fieber eintrat, welches trotz täglicher Chiningaben von 1-2 g 11 Tage die Form einer Febris continua remittens zeigte, darnach in eine Febris quotidiana überging, und uuter Methylen-Gebrauch nach 24 Tagen mit Heilung endete. Die Milz war in den ersten Tagen bereits deutlich geschwollen; mit Ausnahme von heftigen Kopfschmerzen und in der letzten Fieberwoche auftretenden Collaps-Erscheinungen wurden andere bemerkenswerthe Erscheinungen nicht beobachtet. Am 17. Juni Mittags um 12 Ubr wurde das Fingerblut untersucht und eine grosse Anzahl der in Figur 7 gezeichneten Parasiten gefunden. In der letzten Fieberwoche, sowie nach Ablauf des Fiebers war der Blutbefund negativ. 
Tabelle 4.

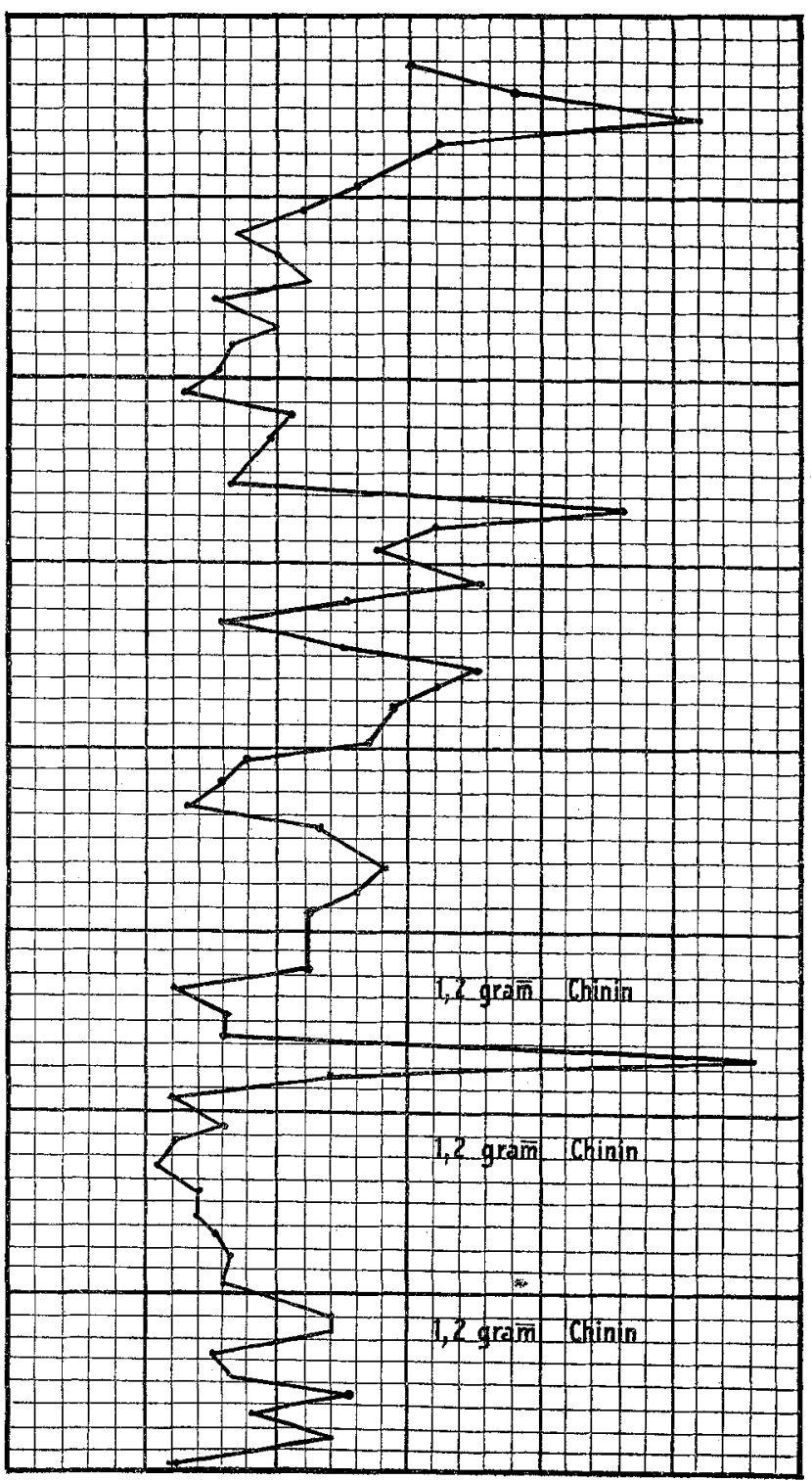


Der vierte der hier vorkommenden Parasiten, den ich ebenfalls zu den Malaria-Erregern zählen möchte, ist der früher in diesem Archiv bereits beschriebene, stark pigmentirte MilzParasit. Ich fand denselben bei einer grossen Zahl fiebernder Beri-Beri-Kranker im Anfang der Erkrankung im Milzblut. Im Fingerblut ist er, wie ich mich später nach der ersten Beschreibung überzeugen konnte, nur in den allerschwersten Fällen zu finden, und zwar nur seine kleinsten Formen. Eine Färbung ist mir nicht gelungen, so dass auch die Art seiner Fortpflanzung nicht mit erforderlicher Sicherheit festzustellen war. Es sind rundliche, stark pigmentirte Gebilde mit weissem Plasma. Das Pigment befindet sich im lebenden Parasiten in starker Bewegung; auf eine nähere Beschreibung verzichte ich und verweise auf das früher Mitgetheilte.

Der Fall, von dem Figur 8 entstammt, war der folgende:

Der Araber Sech Mobamed wurde Mitte Juni ins Krankenhaus wegen leichter Fieberanfälle aufgenommen; er theilte mit, dass das Fieber um 3 Uhr Mittags anfange, bereits 14 Tage anhielte, und dass er in der Fieberzeit, die bis in die Nacht hineinreiche, heftige Schmerzen in den Unterschenkeln verspüre. Milz war vergrössert, Temperatur am 16. Juni Morgens 37,4 , Mittags 5 Uhr $38,1^{\circ}$ O., er bekam jeden Morgen $1 \mathrm{~g}$ Chinin. Nach 6 tägigem Chiningebrauch blieb das Fieber aus; die Temperaturen waren bis zum 22. Juni die folgenden:

$$
\begin{aligned}
& \text { 17. Juni } 37,4-38,4^{\circ} \mathrm{C} \text {, } \\
& \text { 18. " } 37,4-37,9^{\circ} \mathrm{C} \text {., } \\
& \text { 19. } " 37,6-37,9^{\circ} \mathrm{C} \text {., } \\
& \text { 20. } \quad 37,4-37,6^{\circ} \mathrm{C} \text {, } \\
& \text { 21. } \quad 37,2-37,9^{\circ} \mathrm{C} \text {, } \\
& 22 \text {. " } 37,4-38,2^{\circ} \mathrm{O} \text {. }
\end{aligned}
$$

Am 25. Juni klagte er über ein Gefühl von Wärme und Ameisenlaufen in den Unterschenkeln, der Gefühls- und Ortsinn war an beiden Unterschenkeln vollständig aufgehoben, der T'emperatursinn war links vorhanden, reckts für Wärme herabgesetzt, ebenso war der Drucksinn aufgehoben. Patellar-Reflexe rechts herabgesetzt, links deutlicb vorbanden, er füblte Schwere in den unteren Extremitäten und lief etwas steif. Die Bewegungen der Füsse wurden schwerfällig ausgeführt. Puls war beschleunigt, $96 \mathrm{p} . \mathrm{m}$. in Ruhe, Athmung 32 p. m., der Blutdruck wie folgt: Rad. sinistra $230 \mathrm{~mm}$, dextra $180 \mathrm{~mm}$, Femoralis sin. und dextra $230 \mathrm{~mm}$. Es bestand demnach eine Zunabme des Blutdrucks in den untersuchten Arterien. Stuhlgang und Blasen-Function normal, im Urin kein Eiweiss, am Herzen sonst keine krankbaften Erscheinungen. Der Kranke bekam Chinin-Nitroglycerin und 
warme Bäder. Der Puls blieb bis Mitte August leicht beschleunigt, Atbmung normal. Die Temperatur zeigte bisweilen abendliche, leichte Erhebungen bis $37,90 \mathrm{C}$. Die motorischen und sensiblen Störungen zei gten ebenfall Besserung, Ameisenlaufen und Scbmerzen waren viel geringer, auch die Schwere in den Gliedern hatte nachgelassen, bis im August wieder etwas höheres Fieber eintrat und damit auch Kurzathmigkeit, Gefühl von Schwere in den unteren Extremitäten sich zeigten. Die Temperaturen waren die folgenden :

$$
\begin{aligned}
& \text { 16. August } 37,4-38,3^{\circ} \mathrm{C} \text {., } \\
& \text { 17. " } \quad 37,2-38,1^{\circ} \mathrm{C} \text {., } \\
& \text { 18. } \quad 37,1-38,4^{\circ} \mathrm{C} ., \\
& \text { 19. } \quad 37,5-38,5^{\circ} \mathrm{C} .
\end{aligned}
$$

Der Kranke wurde nun mit Methylenblau, 4 mal täglich mit $0,1 \mathrm{~g}$ Nitroglycerin nnd warmen Bädern behandelt. Vom 20. August blieb das Fieber aus; im September und October gingen alle Erscheinungen allmäblich zurück, sodass er Ende October gesund entlassen wurde.

\section{Tafel-Erklär ung.}

Taf. X. Fig. 1. Tertiana duplex. Fingerblut. Nach Ziemann. a) Theilungsformen mit carminvioletten Chromatinkörnern; b) Jugendformen; c) steriler Parasit; d) Lymphocyt; e) rothes Blutkörperchen.

Fig. 2. Fingerblut. Vergr. $1: 1180$. a) grosser pigmentirter Tertiana-Parasit; b) Jugendformen; c) Lymphocyt ; d) rothes Blutkörperchen.

Fig. 3. Fingerblut. Vergr. $1: 1180$. a) Theilungsform des kleinen wenig pigmentirten Quotidian-Parasiten; b) grosse Formen vor derSporulation; $\mathfrak{c}$, Jugendform; d) multinucl. Leukocyt; e) kleiner Lymphocyt; f) grosser Lymphocyt; g) rothes Blutkörperchen.

Fig. 4. Chromatinfärbung nach Ziemann. Fingerblut. Vergr. $1: 1180$. a) Ringelformen mit carmin-violetten ChromatinKörnchen; b) Lymphocyt, c) multinucleärer Leukocyt; d) rothes Blutkörperchen.

Taf. XI. Fig. 5. Malaria - Fingerblut: Vergr. $1: 1180$. a) Sichelformen; b) multinucleär. Leukocyt; c) rothes Blutkörperchen.

Fig. 6. Malaria-Intermittens. Milzblut. Vergr. 1:925. a) Theilungsformen des unpigmentirten Parasiten; b) grosse Formen; c) kleine Formen; d) mittelgrosse Formen; e) Lymphocyt; f) multinucl. Leukocyt; g) roth. Blatkörperch.

Fig, 7. Malaria continua. Fingerblut. Vergr. 1:925. a) kleine Formen der unpigmentirten Parasiten; b) Lymphocyt; c) multinucl. Leukocyt; d) rothes Blutkörperchen.

Fig. 8. Milzblut. Vergr. 1:1180. a) stark grobkörnig pigmentirter grosser Milz-Parasit; b) kleinere Formen; c) mittelgrosse Formen; d) Leukocyt; e) Lymphocyt; f) eosinophile Zelle; g) rothes Blutkörperchen.

Nach Zeichnungen von W. H. Tolsma in Samarang. 